\title{
The Maria asteroid family
}

\author{
S. Aljbaae, ${ }^{1 \star}$ V. Carruba,${ }^{1}$ J. R. Masiero, ${ }^{2}$ R. C. Domingos ${ }^{3}$ and M. Huaman ${ }^{1,4}$ \\ ${ }^{1}$ São Paulo State University (UNESP), School of Natural Sciences and Engineering, Guaratinguetá, SP 12516-410, Brazil \\ ${ }^{2}$ Jet Propulsion Laboratory/Caltech, 4800 Oak Grove Dr., MS 183-601, Pasadena, CA 91109, USA \\ ${ }^{3}$ Universidade Estadual Paulista (UNESP), São João da Boa Vista, CEP 13876-750, SP, Brazil \\ ${ }^{4}$ Observatório Nacional (ON), Rua Gal. Jose Cristino 77, Rio de Janeiro, RJ 20921-400, Brazil
}

Accepted 2017 July 25. Received 2017 July 19; in original form 2017 May 30

\begin{abstract}
The Maria asteroid family is a group of S-type asteroids. Its location adjacent to the left side of the 3J:-1A mean-motion resonances could be the reason for the absence of the left side of the ' $\mathrm{V}$ ' shape in the $(a, 1 / D)$ domain. This family can be considered as a likely source of ordinary chondrite-like material. In this work, we make use of the time dependence of the asymmetric coefficient $A_{S}$ describing the degree of asymmetry of the $C$ distribution of a fictitious Maria family generated with the value of the ejection velocity parameter $V_{\mathrm{EJ}}=35 \mathrm{~m} \mathrm{~s}^{-1}$ to obtain an age estimate of $1750_{+537}^{-231} \mathrm{Myr}$, in good agreement with the family age found in the literature. Analysing the contribution to the near-Earth object (NEO) population, we found that about 7.6 per cent of presently known near-Earth asteroids (NEAs) have orbits similar to asteroids from the Maria family. Only $\sim 1.7$ per cent of our simulated family can stay in NEO space for more than $10 \mathrm{Myr}$, while only five asteroids become NEOs in the last $500 \mathrm{Myr}$ of the simulation.
\end{abstract}

Key words: gravitation - celestial mechanics - minor planets, asteroids: general.

\section{INTRODUCTION}

Among asteroid families, the Maria group is peculiar because of its location adjacent to the left side of the $3 \mathrm{~J}:-1 \mathrm{~A}$ mean-motion resonances with Jupiter (hereafter MMRs). This seems very likely to be responsible for the absence of the left side of its $\mathrm{V}$ shape (Spoto, Milani \& Knežević 2015). The Maria family can be considered as a likely source of ordinary chondrite-like material and a possible parent body of the giant near-Earth asteroids 433 Eros and 1036 Ganymed (Zappalà et al. 1997; Zappalà \& Cellino 2001). The position of the Maria family (near the 3J:-1A MMR) increases its possible contribution to near-Earth material (Morbidelli, Thomas \& Moons 1995). Our selected family is a typical group 3 Gyr old, as estimated by Nesvorný et al. (2005) and Brož et al. (2013). Considering Maria as a one-sided family, for which one of the two sides of the $\mathrm{V}$ shape is non-identifiable, Spoto et al. (2015) estimated the age of this family as $1.932 \mp 0.422$ Gyr. Carruba \& Nesvorný (2016) estimated an age of $\sim 1.950$ Gyr using equation (1) of Nesvorny, Broz \& Carruba (2015). Identifing the footprints of the Yarkovsky-O'KeefeRadzievskii-Paddack (YORP) effect, Paolicchi \& Knežević (2016) also gave an independent estimate of the age of the Maria family by using the slopes of the V plot; they found an age of $1932 \mathrm{Myr}$.

According to Nesvorny et al. (2015), 2940 members of the Maria family (Family Identification Number FIN = 506) were identified using the hierarchical clustering method (HCM: Zappala et al. 1994;

* E-mail: safwan.aljbaae@gmail.com
Bendjoya \& Zappalà 2002) with a cut-off distance of $60 \mathrm{~m} \mathrm{~s}^{-1}$. Their semi-major axis values (a) range from 2.521970-2.720380 au, their eccentricities $(e)$ from $0.127810-0.127810$ and their sines of inclination $(\sin (i))$ from $0.235980-0.266530$. Also, Milani et al. (2014) identified 2742 members of the Maria family (2320 numbered and 422 multi-opposition asteroids), with proper orbital elements varying from $2.52277-2.67311$ au in $a, 0.06679-0.12828$ in $e$ and $0.23080-0.26864$ in $\sin (i)$, considering $90 \mathrm{~m} \mathrm{~s}^{-1}$ as the critical quasi-random level (QRL). The full black dots in Fig. 1 identify the orbital location of the Maria HCM family members with a dense cluster at its core, as identified in Nesvorny et al. (2015). (170) Maria is the lowest numbered member in the family. The full orange dots denote the Maria family members as identified in Milani et al. (2014). The Maria family identified in Milani et al. (2014) is 6.73 per cent smaller, despite the presence of numbered and multi-opposition objects.

In this work, we will use the data from Nesvorny et al. (2015) to investigate the orbital evolution of members of the Maria family. First, in Section 2, we revise current knowledge of the taxonomy and physical properties of asteroids in the family region. Then, the local dynamics in the family region are investigated in Section 3. The methods of Yarkovsky isolines and the Yarko-YORP Monte Carlo approach are used in Section 4 to obtain estimates for the Maria family's age. In Section 5, we deal with the local dynamical evolution of the Maria family to improve the age estimation. We also analyse the possible contribution to the nearEarth object (NEO) population. Finally, our conclusions are given in Section 6 . 

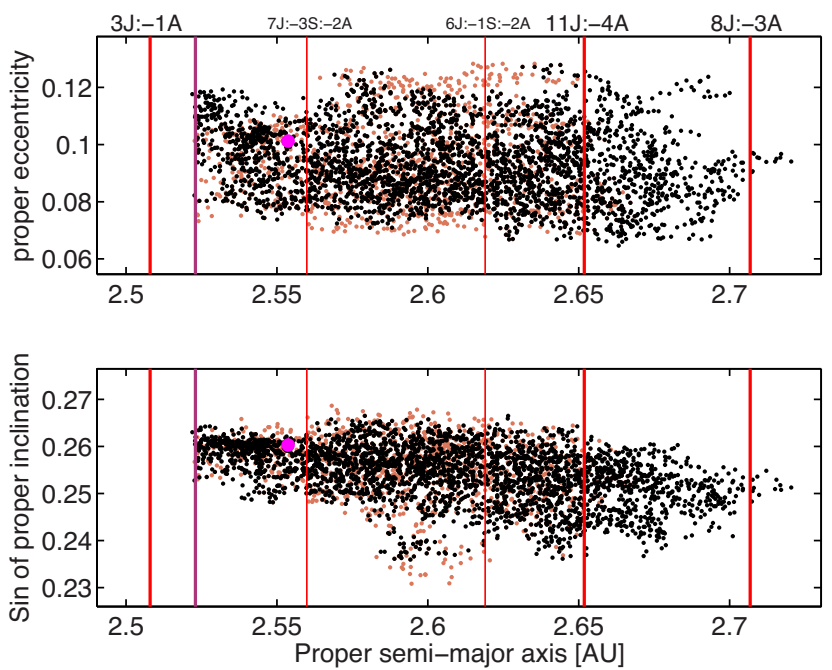

Figure 1. Top panel: a projection in the $(a, e)$ domain of 2940 members of the Maria family identified in Nesvorny et al. (2015) (full black dots) and 2742 members identified in Milani et al. (2014) (full orange black dots). Bottom panel: a projection in the $(a, \sin (i))$ domain of the same asteroids. Vertical heavy and light red lines display the main two- and three-body mean-motion resonances. The indigo line displays the chaotic layer near the 3J:-1A MMR (zone within $0.015 \mathrm{au}$ ). The position of (170) Maria itself is shown as a magenta full dot.
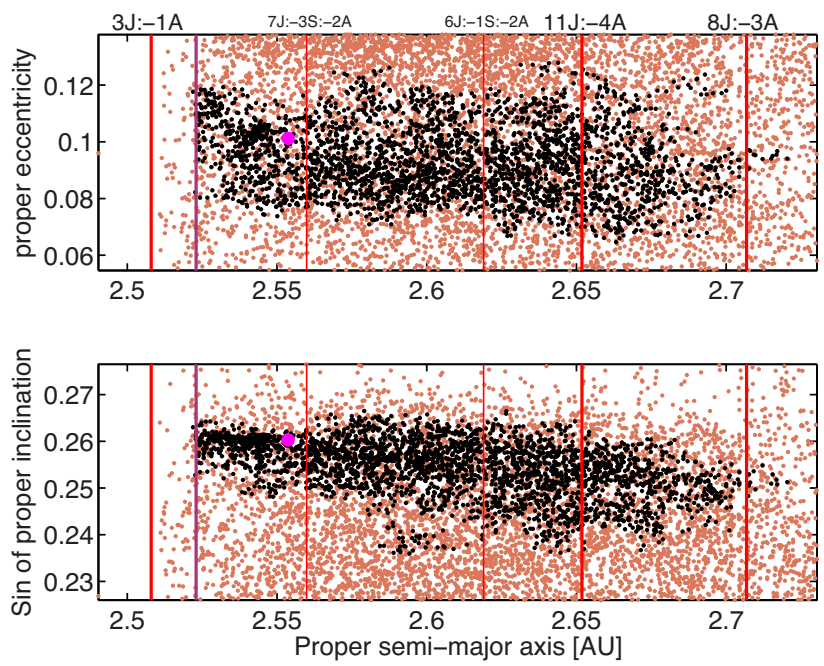

Figure 2. Top panel: a projection in the $(a, e)$ domain of 8146 AstDyS asteroids in the region of the Maria family (orange dots) and 2940 members of the Maria HCM cluster (black dots). Bottom panel: a projection in the ( $a$, $\sin (i))$ domain of the same asteroids. See the figure legend for the meaning of the full dot symbols.

\section{PHYSICAL PROPERTIES}

As a preliminary step in our analysis, we identified the local background of the Maria family, selecting 8146 asteroids from the Asteroids Dynamic Site (AstDyS) ${ }^{1}$ data base with minimum and maximum values within \pm 0.02 of the $a, e$ and $\sin (i)$ of the members of the Maria HCM family. The minimum values of $a$ were fixed at 2.498, 0.01 from the semimajor axis of the centre of the 3J:-1A MMR (see Fig. 2). The local background limits were delimited according to the average of the orbital mobility caused by close encounters with

\footnotetext{
${ }^{1}$ http://hamilton.dm.unipi.it/astdys/, accessed on March 2016.
}

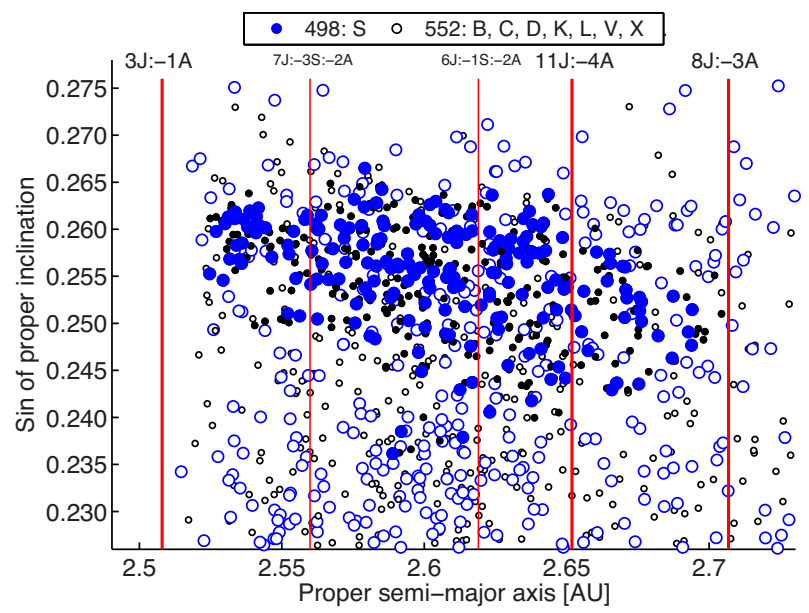

Figure 3. An $(a, \sin (i))$ projection of the 1050 asteroids in the Maria region with taxonomic information from the SDSS-MOC4 data base. The circles correspond to asteroids in the background, while the full dots correspond to asteroids in the Maria HCM group. See the figure legend for the meaning of the symbols.

massive asteroids over the family maximum estimated age (Carruba et al. 2013).

We then examined current knowledge regarding the taxonomical properties and albedo information for members in the Maria region. The fourth release of the Sloan Digital Sky Survey Moving Object Catalog (SDSS-MOC4: ${ }^{2}$ Ivezić et al. 2001) listed 1050 asteroids in Maria's background with photometric data, 432 of which are in the HCM group. Using these data, we obtained the taxonomical information with the method described in DeMeo \& Carry (2013). In the background, we found 47.4 percent S-type, 36.3 per cent L-type and a 16.3 percent $\operatorname{mix}$ of $\mathrm{A}, \mathrm{C}, \mathrm{D}, \mathrm{K}$ and X-type. 45.6 per cent of members in the HCM group are classified as Stype, 37 percent as L-type and there is a 7.4 percent mix of C, D, $\mathrm{K}$ and $\mathrm{X}$-type. Our results are summarized in Fig. 3. Asteroids in the background of the Maria family are presented as circles and asteroids in the Maria HCM group are presented as full dots.

We also revised the albedo estimates from the Wide-field Infrared Survey Explorer (WISE) and Near-Earth Object WISE ${ }^{3}$ (Masiero et al. 2011; Mainzer et al. 2016). There are 674 asteroids in the Maria HCM group with geometric albedo. 501 of them do not have taxonomic information. About 65 per cent of those last albedo values vary between 0.12 and 0.30 and are possibly associated with an S-type. Fig. 4 displays our results for these albedos. The mean and median albedo found in the group are 0.2609 and 0.2598 , respectively.

As in Aljbaae et al. (2017), we also evaluated the effective diameters $(D)$ of members in Maria HCM group. The diameters obtained vary between 0.764 and $50.322 \mathrm{~km}$. Considering a spherical shape for each asteroid with uniform density, the value of which is different for different taxonomic classes (DeMeo \& Carry 2013), the total mass of the family is estimated as $0.115 \times 10^{19} \mathrm{~kg}$. Assuming that the objects on the left side of the family were lost in the 3J:-1A MMR, our family probably originated from the break-up of an S-type asteroid with a diameter $D \approx 120 \mathrm{~km}$, which is in good agreement with the size for the parent body estimated in Brož et al. (2013) using the Durda et al. (2007) method.

\footnotetext{
${ }^{2}$ http://faculty.washington.edu/ivezic/sdssmoc/sdssmoc.html

${ }^{3} \mathrm{http} / / /$ wise2.ipac.caltech.edu/staff/bauer/NEOWISE_pass1/
} 


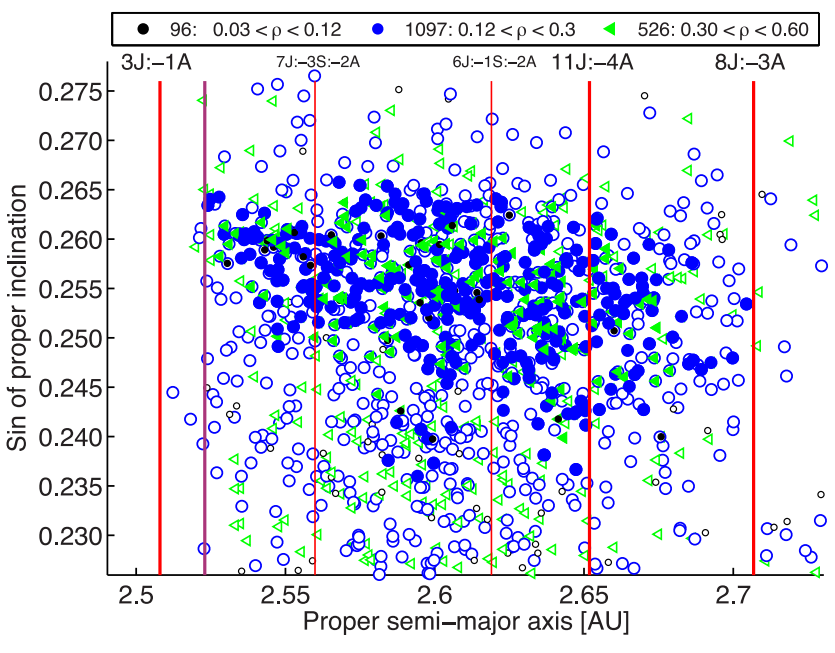

Figure 4. An $(a, \sin (i))$ projection of the 501 bodies in the Maria region with albedo information from the WISE data set. See the figure legend for the meaning of other symbols

\section{DYNAMICAL MAP}

In order to investigate the local dynamics, we constructed a dynamical map for the region of Maria by obtaining the synthetic proper elements for 24000 massless test particles placed on a grid 80 by 300 in the $(a, \sin (i))$ plane and for 10400 particles within a grid 80 by 130 in the $(a, e)$ plane. Using the SWIFT_MVFS symplectic integrator from the SWIFT package (Levison \& Duncan 1994), modified by Brož (1999), we integrated the particles over $20 \mathrm{Myr}$ under the influence of all planets, neglecting self-interactions between asteroids, which could act as a source to increase orbital perturbation. The initial values of the eccentricity $e$ for the simulations in the $(a, \sin (i))$ plane, the inclination for the simulations in the $(a, e)$ plane, the longitude of the ascending node $\Omega$, the argument of the pericentre $\omega$ and the true anomaly $\lambda$ for each test particle were fixed at those of (170) Maria at a modified Julian date of 57200. Synthetic proper elements and frequencies were determined using the approach discussed in Carruba (2010). Fig. 5 displays our results. The top panel presents the dynamical map in the proper $(a, \sin (i))$ plane; the region devoid of particles denotes the strongly unstable region at the $3 \mathrm{~J}:-1 \mathrm{~A}$ MMR. Black full dots identify the values of $a$ and $\sin (i)$ of each particle. Here, we just indicate the role of two nonlinear secular resonances, which affect the dynamical evolution of a significant number of asteroids in the core asteroid belt. The (s-s6)-(g5-g6) resonance is presented as amber full points; this resonance was identified by Milani \& Knezevic (1994) as an important source of error in the determination of proper elements. The $2(\mathrm{~g}-\mathrm{g} 6)+(\mathrm{s}-\mathrm{s} 6)$ resonance is presented as green full points; this resonance can prevent a reliable small family identification (Zappala et al. 1994). The bottom panel presents the dynamical map in proper $(a, e)$. Here, the $2(\mathrm{~g}-\mathrm{g} 6)+(\mathrm{s}-\mathrm{s} 6)$ resonance is also presented using green full points. The $\mathrm{g}-\mathrm{s}$ type $\mathrm{g}-2 \mathrm{~g} 6+\mathrm{g} 5-\mathrm{s}-\mathrm{s} 6$ resonance is presented as amber full points. One can clearly notice the lack of objects around the $\mathrm{g}-2 \mathrm{~g} 6+\mathrm{g} 5-\mathrm{s}-\mathrm{s} 6$ resonance and the concentration of objects at very small eccentricities. For a qualitative comparison with the map, the proper elements of real objects in the Maria HCM group are plotted as blue full dots. Carruba \& Michtchenko (2007a), Carruba, Michtchenko \& Lazzaro (2007b) and Carruba (2010) analysed the dynamics of this region in more detail; the interested reader can find more information in those articles.
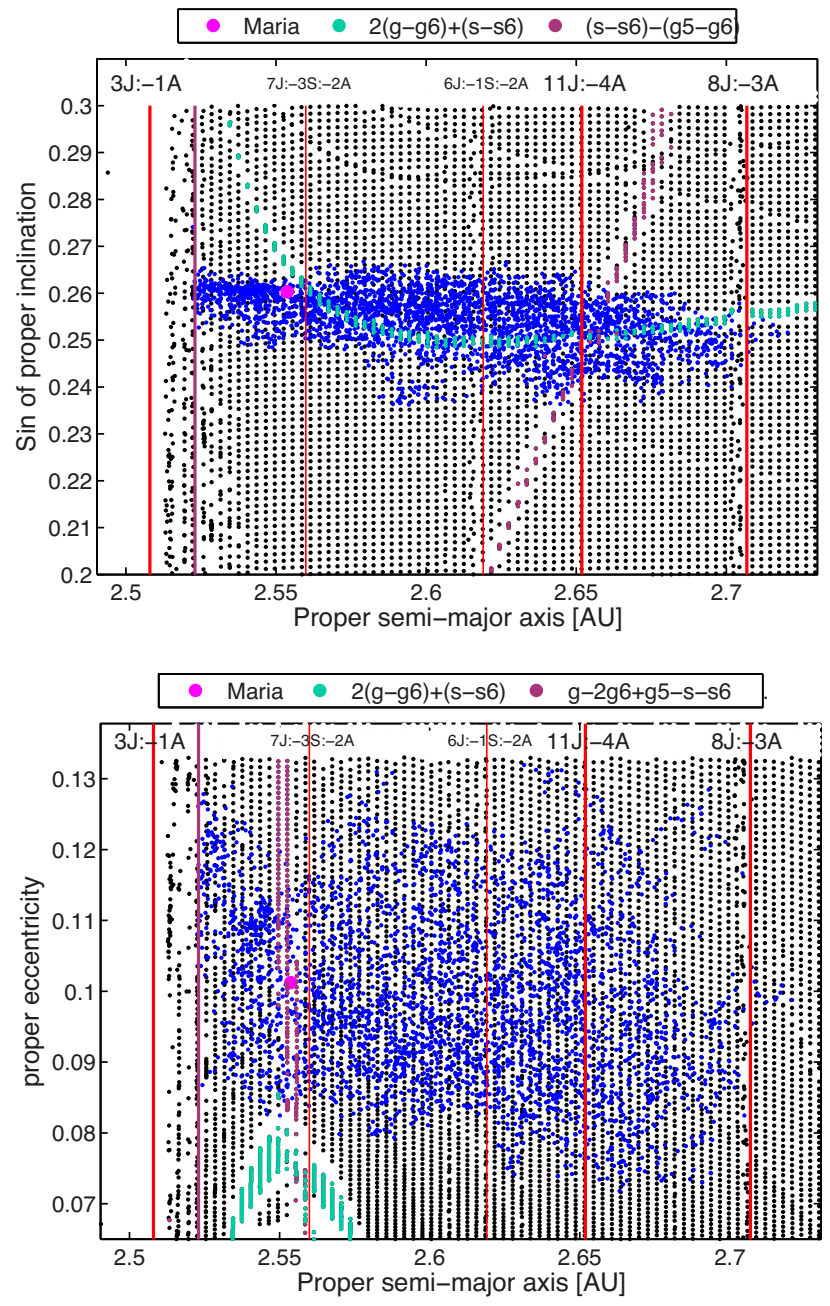

Figure 5. Proper semi-major axis versus sin of proper inclination (top panel) and proper eccentricity distribution (bottom panel) for objects in the region of the Maria family. Black dots display the proper elements of test particles. The real objects in the Maria HCM group are presented as blue full dots. See the figure legend for the meaning of the full dot symbols.

\section{CHRONOLOGY}

We used the Yarkovsky isolines method to obtain a preliminary estimate of the family's age. Our results are displayed in Fig. 6. We computed the isolines for $T=1932 \mathrm{Myr}$, the estimated age according to Spoto et al. (2015), and for $T=3000 \mathrm{Myr}$ according to Nesvorný et al. (2005) and Brož et al. (2013). In this analysis, we used the parameters describing the Yarkovsky force for S-types listed in Brož et al. (2013). Only three asteroids are classified as dynamical interlopers (id: 660, 4860 and 15494), shown in Fig. 6 as black full dots. No physical data on these interlopers were found, thus it is difficult to know whether they are also physical interlopers.

In order to refine the family age estimation previously obtained, we followed the work of Carruba et al. (2014a), Carruba, Aljbaae \& Souami (2014b) and Carruba et al. (2015), analysing the semimajor axis evolution of a fictitious distribution of asteroids, evolved for different values of the ejection velocity parameter $\left(V_{\mathrm{EJ}}\right)$ under the influence of Yarkovsky diurnal and seasonal effects. We used the Monte Carlo method of Vokrouhlický et al. (2006a,b,c). This method was modified by (Carruba et al. 2015) to include the stochastic YORP force and changes in values of the past solar luminosity. We first obtained a histogram of a $C$-target function (Fig. 7) through 


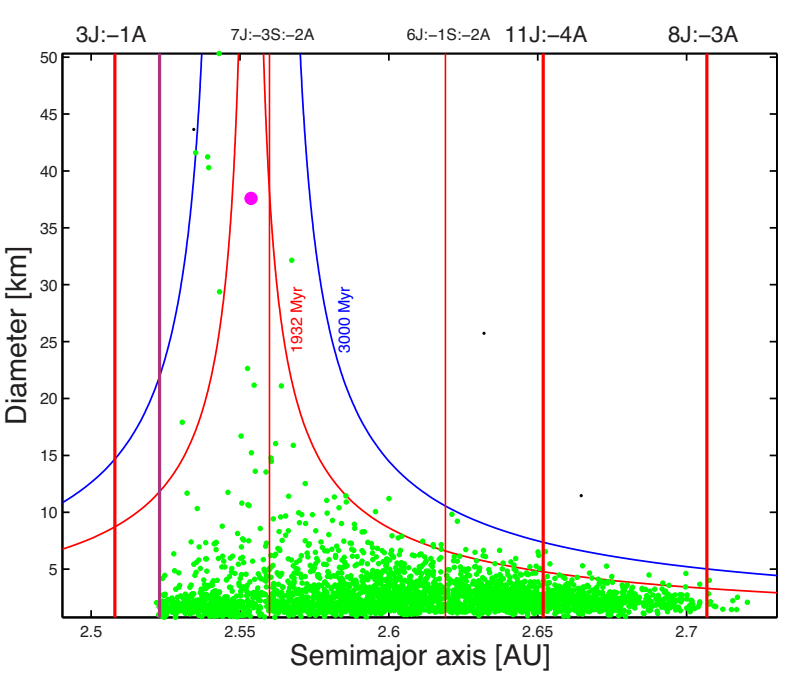

Figure 6. The time-scale isolines of the Yarkovsky effect for the Maria family with respect to the current location of (170) Maria. The red and blue lines correspond to $T=1932$ and $3000 \mathrm{Myr}$, respectively. The green full dots denote the real objects in the HCM group. The position of 170 Maria itself is shown as a magenta full dot.

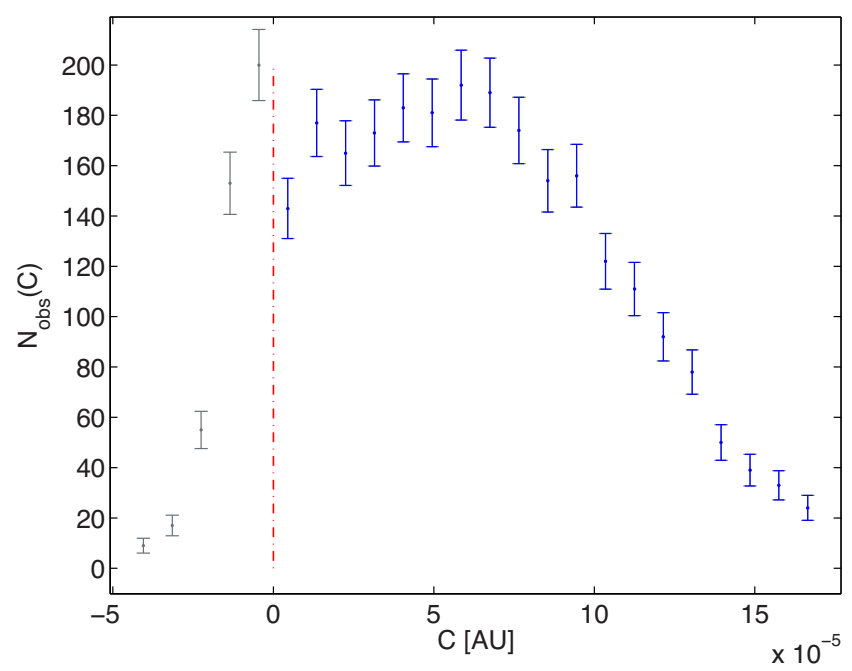

Figure 7. A histogram of values of the $C$ target function from equation (1) for the Maria family.

the equation

$0.2 H=\log _{10}\left(\frac{\Delta a}{C}\right)$,

where $H$ is the absolute magnitude of an asteroid and $\Delta a=a-a_{0}$ its distance from the central value of the semi-major axis $\left(a_{0}\right)$, assumed to be the current location of (170) Maria. 24 intervals are used, starting at $-4.5 \times 10^{-5}$ with a step of $9.0 \times 10^{-6}$ au. In our analysis, we neglected negative $C$ values (five grey lines in Fig. 7), so as to concentrate on the right side of the family. Thus, our distribution has $17(19-2)$ degrees of freedom. To avoid problems caused by uncertainty in the orbital position of Maria, we computed the average of the $C$ distribution for the family, obtained as a mean of five values, corresponding to values of $C$ in the range $\pm 0.05 \mathrm{au}$ (maximum drift caused by the Yarkovsky effect) near the position of Maria.

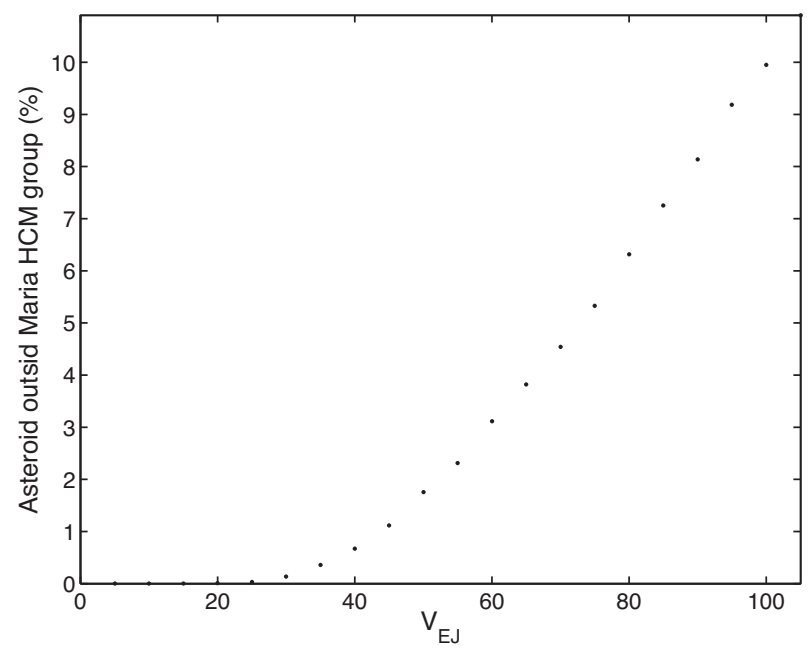

Figure 8. Percentile of of particles with values of $\sin (i)$ outside the range of values currently observed in the Maria HCM group.

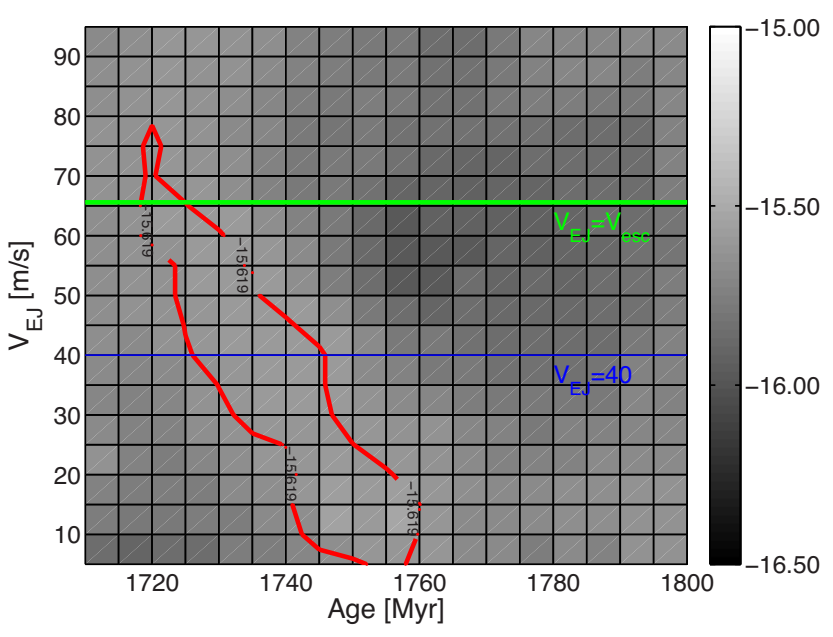

Figure 9. Values of the $\psi \Delta C$ function in the (age, $V$ ) plane. The horizontal blue lines display the value of the escape velocity $\left(V_{\mathrm{esc}}=65 \mathrm{~m} \mathrm{~s}^{-1}\right)$ and the maximum value of the ejection velocity $\left(40 \mathrm{~m} \mathrm{~s}^{-1}\right)$.

As was previously mentioned, the Maria family probably originated from an S-type parent body $120 \mathrm{~km}$ in diameter, which yields an estimate of $65 \mathrm{~m} \mathrm{~s}^{-1}$ for the escape velocity $\left(V_{\mathrm{esc}}\right)$. Assuming that $V_{\mathrm{EJ}}$ does not generally exceed $1.5 V_{\text {esc }}$ (Carruba 2016), the maximum values of $V_{\mathrm{EJ}}$ considered here will be $100 \mathrm{~m} \mathrm{~s}^{-1}$. We checked the number of surviving particles in the Maria region as a function of time. Fig. 8 shows the percentile of particles with values of $\sin (i)$ outside the range of values currently observed (from $0.2360-0.2665)$. This implies that $V_{\mathrm{EJ}}$ was most likely smaller than $40 \mathrm{~m} \mathrm{~s}^{-1}$.

Assuming that half of the Maria family was lost in the 3J:1A MMR, fictitious families, obtained with the approach of Vokrouhlický et al. (2006b), were simulated under the influence of Yarkovsky and YORP forces, producing distributions of the $C$ target function for different values of $V_{\mathrm{EJ}}$. The age and ejection velocity parameter that fit the observed data best can be obtained by minimizing a $\chi^{2}$-like function (Carruba et al. 2014b). Our results are shown in Fig. 9. The red line corresponding to $\Psi \Delta C=15.62$ yields a probability of 68.27 per cent that the simulated and observed distributions are compatible. We found an age of $1735_{-17}^{+25} \mathrm{Myr}$ and 


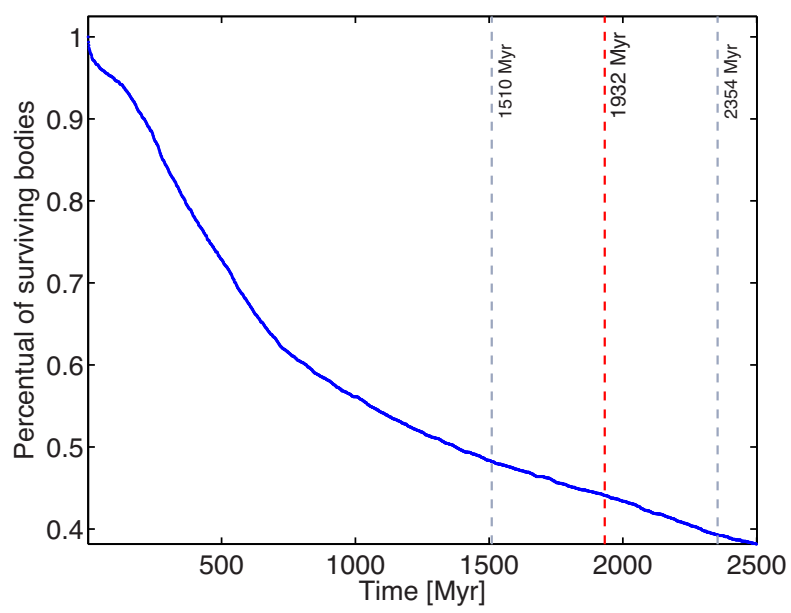

Figure 10. Percentile of surviving particles in the Maria region as a function of time. Vertical dashed lines display the estimated age of the family.

an ejection velocity parameter of $35_{-30}^{+45} \mathrm{~m} \mathrm{~s}^{-1}$. Our age estimation is compatible with the age of Spoto et al. (2015).

\section{DYNAMICAL EVOLUTION OF THE MARIA FAMILY}

In this section, we investigate how the orbital elements of Maria family members evolve with time after the parent body's break-up, by simulating the initial conditions of members of a fictitious Maria family generated from the real asteroids in the Maria HCM group with absolute magnitudes $H<15$, with the value $V_{\mathrm{EJ}}=35 \mathrm{~m} \mathrm{~s}^{-1}$. We used the SWIFT_RMVSY code from the SWIFT package (Levison \& Duncan 1994), modified by Brož (1999), to forward-integrate 2340 massless particles over $2500 \mathrm{Myr}$ under the gravitational perturbations of all planets, with a time step of $20 \mathrm{~d}$. We used two initial spin axes forming angles of $0^{\circ}$ and $180^{\circ}$ with the orbital angular momentum, so as to consider the maximum drift speeds of the diurnal Yarkovsky effect. As in the previous section, we used the thermal parameters for $\mathrm{S}$ taxonomic types of asteroids listed in Brož et al. (2013): $\rho_{\text {surf }}=2500$ and $\rho_{\text {bulk }}=1500 \mathrm{~kg} \mathrm{~m}^{-3}$ for the surface and bulk densities, $C=680 \mathrm{~J} \mathrm{~kg}^{-1} \mathrm{~K}^{-1}$ for the thermal capacity, $A=0.1$ for the Bond albedo, $\epsilon_{\mathrm{IR}}=0.9$ for the thermal emissivity and $K=0.001 \mathrm{~W} \mathrm{~m}^{-1} \mathrm{~K}^{-1}$ for the surface thermal conductivity. We calculated the synthetic proper elements for each test with the approach described in Carruba (2010). Fig. 10 presents the number of particles that survived the full integration as a function of time. The vertical dashed lines are associated with the estimated family age and uncertainty. Less than 50 percent of the original family members remained in the region, because of the interaction with the 3J:-1A MMR.

According to Carruba \& Nesvorný (2016), the Maria family is a platykurtic family, with estimated value of the kurtosis of the $v_{W}$ component of the ejection velocity field $\gamma_{2}\left(v_{W}\right)=-0.17$. Thus, it is not easy to apply the method based on the time behaviour of $\gamma_{2}\left(v_{W}\right)$ (Carruba \& Nesvorný 2016; Carruba et al. 2016; Carruba, Novaković \& Aljbaae 2017; Aljbaae et al. 2017). In this work, we used the asymmetry coefficient $\left(A_{S}\right)$ recently introduced in Aljbaae et al. (2017) to analyse the degree of asymmetry of the Maria family. This coefficient was defined as

$A_{S}=\frac{1}{N_{\text {ast }} \cdot N_{\text {int }}} \sum_{1}^{N_{\text {int }}}\left(N_{\text {pos }}(i)-N_{\text {neg }}(i)\right)$,

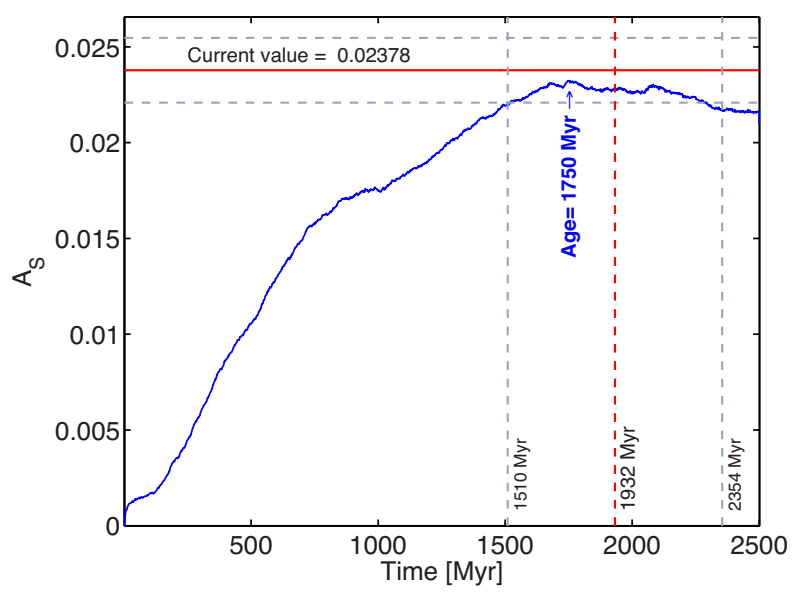

Figure 11. Asymmetry coefficient of the target function. The horizontal lines display the current value of $A_{S}$ and its error, obtained with standard error propagation formulae.

where $N_{\text {ast }}$ is the number of objects in the simulated family, $N_{\text {int }}$ is the number of positive intervals in the $C$ distribution and $N_{\text {pos }}(i)$ and $N_{\text {neg }}(i)$ are the number of asteroids in the positive and negative $i$ th bin of the distribution. As in the case of the Rafita family, the current distribution of $C$ values presented in Fig. 7 is considerably asymmetric $\left(A_{S}=0.024 \mp 0.0017\right)$. In Fig. 11 , we report the time behaviour of the values of $A_{S}$ for our simulated family as a function of time. Our results showed good agreement with the age estimated by Spoto et al. (2015), Paolicchi \& Knežević (2016) and Carruba \& Nesvorný (2016). The figure shows two different peaks: one after $1750_{+537}^{-231}$ Myr and the other after $2100_{+590}^{-254} \mathrm{Myr}$ of the parent body's break-up, which could be related to some numerical artefact in the SWIFT package. However, the two peaks are inside the allowed age error range and the first one is closer to the current value of $A_{S}$ than the second peak.

The contribution to the NEO population from the Maria family is also estimated in this work, selecting all objects with perihelia $q<1.3$ au, which would be classified as NEOs (Masiero et al. 2015). We found that 1205 particles ( $~ 7.6$ per cent of the 15891 presently known near-Earth asteroids (NEAs)) moved into NEO space during the simulation. Fig. 12 shows the orbits of the 15891 currently known NEAs ${ }^{4}$ (white points) and the probability densities of the orbital parameters for all particles from the Maria family that become NEOs. Most of these particles are observed at a semimajor axis range between 2.4 and $2.6 \mathrm{au}$, an inclination between $18.7^{\circ}$ and $36.9^{\circ}$ and eccentricities between 0.47 and 0.72 . Only 81 particles $(\sim 1.7$ percent of our simulated family) remained in NEO space for more than $10 \mathrm{Myr}$ : the majority of these objects arrived shortly after the formation of the family; only five of them became NEOs in the last $500 \mathrm{Myr}$ of the simulation. In Fig. 13, we present the orbital element evolution of these particles, where each colour represents an object and each point represents the instantaneous orbital elements every $10 \mathrm{kyr}$. As already mentioned, the Maria family is a possible parent body of two giant NEAs (433 Eros and 1036 Ganymed). Among the orbits of the particles at the end of our simulation, no orbit was found close to Eros, while the closest orbit to Ganymed has a semimajor axis of 2.6559 au, eccentricity of 0.5459 and inclination of $23.1732^{\circ}$.

\footnotetext{
${ }^{4}$ http://www.minorplanetcenter.net/iau/MPCORB/NEA.txt
} 

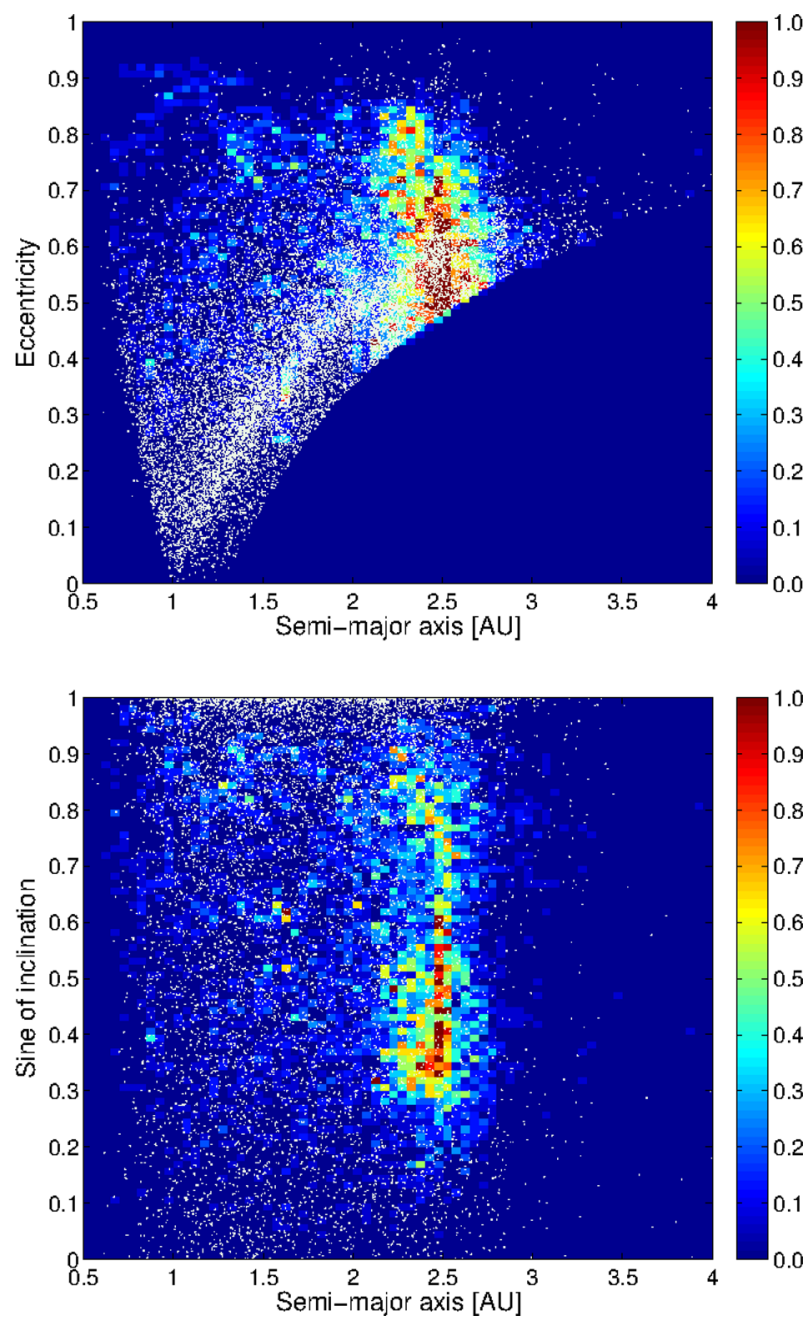

Figure 12. Density maps of orbital elements of NEAs originating from the Maria family. The colour code is associated with the probability of visiting the given region. The white points present the currently known NEAs.

We also conducted independent simulations using a shorter time step of $3 \mathrm{~d}$ to constrain the behaviour of objects better once they enter the NEO region. As shown in Fig. 14, we find that the overall probability map structure remains the same, although an extension in the probability cloud to lower semi-major axes and moderate eccentricities is observed that is absent from the other simulations. For families like Maria that primarily enter near-Earth space via a mean-motion resonance and are quickly removed, longer time-step integrations are sufficient for establishing the baseline probability location for NEOs from this family.

\section{CONCLUSION}

The analysis carried out in this article was performed based on the assumption that the objects on the left side of the Maria family were lost in the 3J:-1A MMR. We first revised current knowledge of taxonomical properties and albedo information for the Maria region. We found that Maria probably originated from the break-up of an S-type asteroid with a diameter $D \approx 120 \mathrm{~km}$. The family interacts with the (s-s6)-(g5-g6) and g-2g6+g5-s-s6 resonances. We then eliminate possible dynamical interlopers using the method of Yarkovsky isolines. The Monte Carlo method was used to analyse the semi-major axis evolution of several fictitious asteroid families
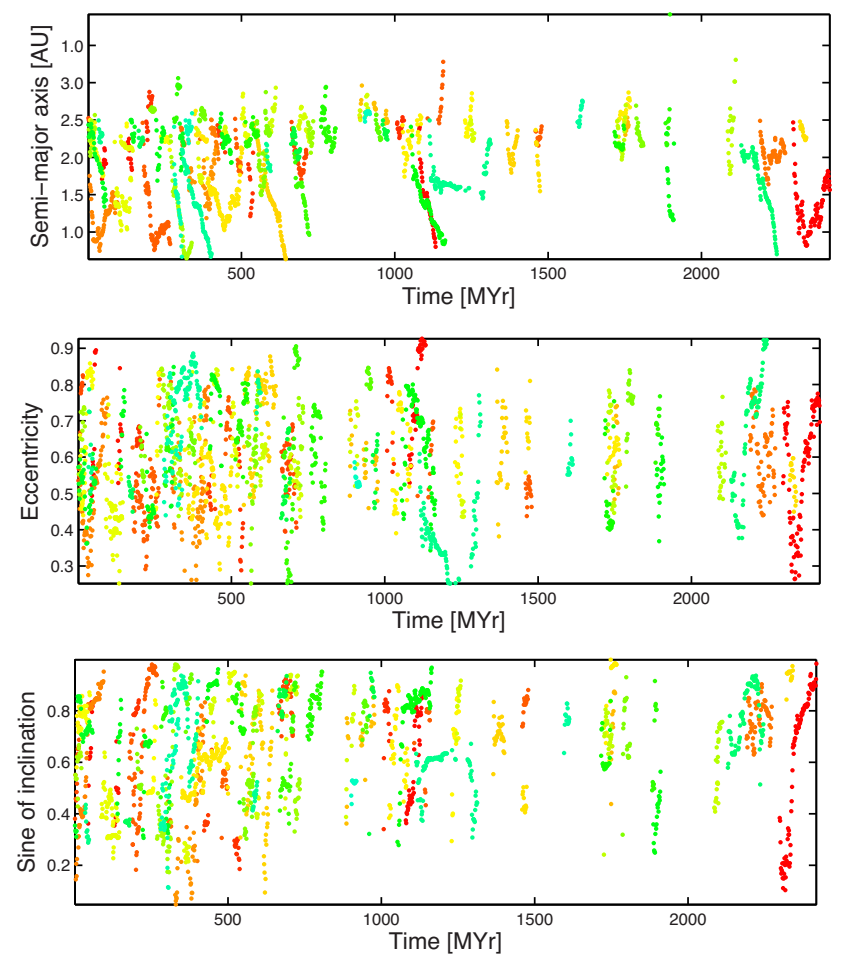

Figure 13. The particles from the Maria family, separated by colours, that remain in NEO space for more than $10 \mathrm{Myr}$.

under the influence of the Yarkovsky and YORP effects. We find an age of $1735_{-17}^{+25} \mathrm{Myr}$ and a value of the ejection velocity parameter of $35_{-30}^{+45} \mathrm{~m} \mathrm{~s}^{-1}$. We then studied the dynamical evolution of a fictitious Maria family, simulating the initial conditions of particles with absolute magnitudes $H<15$ after the break-up of the parent body. Using the method recently introduced in Aljbaae et al. (2017) based on the time dependence of the coefficient $A_{S}$ describing the asymmetry of the $C$ distribution, we obtained an age estimation of $1750_{+537}^{-231}$, in good agreement with previous estimates of the Maria family age obtained by Spoto et al. (2015) and Carruba \& Nesvorný (2016). We studied the possible contribution of the Maria family to the NEO population: $\sim 7.6$ per cent of presently known NEAs have orbits similar to asteroids from the Maria family. Only $\sim 1.7$ per cent of our simulated family can stay in NEO space for more than $10 \mathrm{Myr}$; five objects become NEOs in the last $500 \mathrm{Myr}$ of the simulation.

Overall, using the new method to date the age of incomplete asteroid families introduced in Aljbaae et al. (2017), our results refined previous estimates of the Maria family age, with a new estimate of $1750_{+537}^{-231}$ Myr. This age is in agreement with an independent method based on Monte Carlo simulations of the Yarkovsky and YORP dynamical evolution of family members. We also found that the Maria family could potentially contribute to the current NEO population via the mean-motion resonance $3 \mathrm{~J}$ :-1A.

\section{ACKNOWLEDGEMENTS}

We are grateful to the reviewer of this article, Dr Federica Spoto, for comments and suggestions that greatly improved the quality of this work. The authors also thank the São Paulo State Science Foundation (FAPESP) (Grants 13/15357-1 and 14/06762-2) and the Brazilian National Research Council (CNPq, grants 305453/2011-4 and 310317/2016-9) for their generous support of this work. 

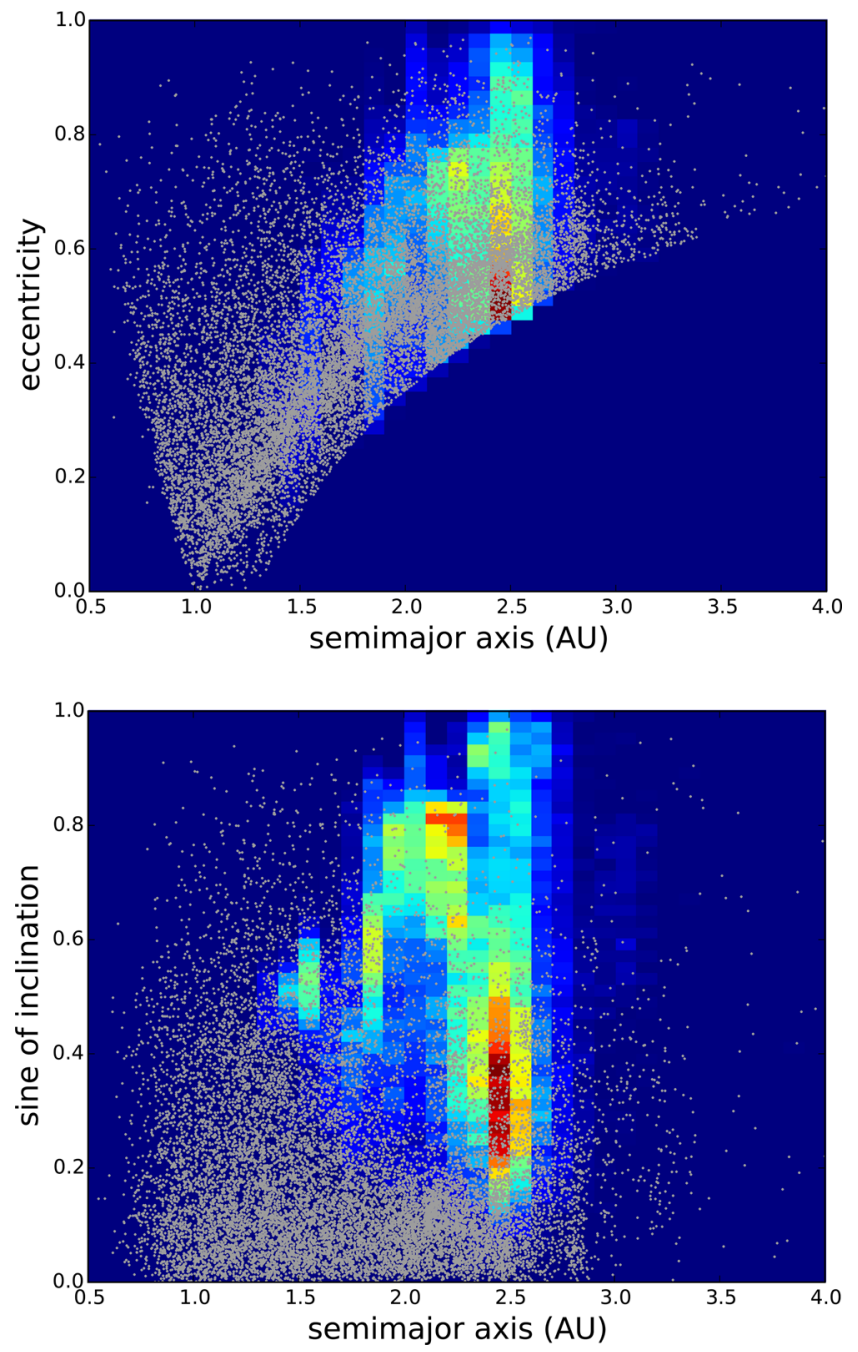

Figure 14. Density maps of orbital elements of NEAs originating from the Maria family using a time step of $3 \mathrm{~d}$.

\section{REFERENCES}

Aljbaae S., Carruba V., Masiero J. R., Domingos R. C., Huaman M., 2017, MNRAS, 467, 1

Bendjoya P., Zappalà V., 2002, in Bottke W. F. Jr., Cellino A., Paolicchi P., Binzel R. P., eds, Asteroid Family Identification. Asteroids III, p. 613 Brož M., 1999, Master's thesis, Charles Univ., Prague

Brož M., Morbidelli A., Bottke W. F., Rozehnal J., Vokrouhlický D., Nesvorný D., 2013, A\&A, 551, A117
Carruba V., Michtchenko T. A., 2007, A\&A, 475, 1145

Carruba V., Michtchenko T. A., Lazzaro D., 2007, A\&A, 473, 967

Carruba V., 2010, MNRAS, 408, 580

Carruba V., 2016, MNRAS, 461, 1605

Carruba V., Nesvorný D., 2016, MNRAS, 457, 1332

Carruba V., Huaman M., Domingos R. C., Roig F., 2013, A\&A, 550, A85

Carruba V., Domingos R. C., Huaman M. E., Santos C. R. D., Souami D., 2014a, MNRAS, 437, 2279

Carruba V., Aljbaae S., Souami D., 2014b, ApJ, 792, 46

Carruba V., Nesvorný D., Aljbaae S., Huaman M. E., 2015, MNRAS, 451, 244

Carruba V., Domingos R. C., Aljbaae S., Huaman M., 2016, MNRAS, 463, 705

Carruba V., Novaković B., Aljbaae S., 2017, MNRAS, 465, 4099

DeMeo F. E., Carry B., 2013, Icarus, 226, 723

Durda D. D., Bottke W. F., Nesvorný D., Enke B. L., Merline W. J., Asphaug E., Richardson D. C., 2007, Icarus, 186, 498

Ivezić Ž. et al., 2001, AJ, 122, 2749

Levison H. F., Duncan M. J., 1994, Icarus, 108, 18

Mainzer A. K. et al., 2016, VizieR Online Data Catalog, 178,

Masiero J. R. et al., 2011, ApJ, 741, 68

Masiero J. R., Carruba V., Mainzer A., Bauer J. M., Nugent C., 2015, ApJ, 809,179

Milani A., Knezevic Z., 1994, Icarus, 107, 219

Milani A., Cellino A., Knežević Z., Novaković B., Spoto F., Paolicchi P., 2014, Icarus, 239, 46

Morbidelli A., Thomas F., Moons M., 1995, Icarus, 118, 322

Nesvorný D., Jedicke R., Whiteley R. J., Ivezić Ž., 2005, Icarus, 173, 132

Nesvorny D., Broz M., Carruba V., 2015, in Michel P., DeMeo F. E., Bottke W., eds, Asteroid IV. Univ. Arizona Press, Tucson, AZ

Paolicchi P., Knežević Z., 2016, Icarus, 274, 314

Spoto F., Milani A., Knežević Z., 2015, Icarus, 257, 275

Vokrouhlický D., Brož M., Morbidelli A., Bottke W. F., Nesvorný D., Lazzaro D., Rivkin A. S., 2006a, Icarus, 182, 92

Vokrouhlický D., Brož M., Bottke W. F., Nesvorný D., Morbidelli A., 2006b, Icarus, 182, 118

Vokrouhlický D., Brož M., Bottke W. F., Nesvorný D., Morbidelli A., 2006c, Icarus, 183,349

Zappalà V., Cellino A., 2001, Astrophysics and Space Science Library, Vol. 261, p. 303

Zappala V., Cellino A., Farinella P., Knezevic Z., 1990, AJ, 100, 2030

Zappala V., Cellino A., Farinella P., Milani A., 1994, AJ, 107, 772

Zappalà V., Cellino A., Di Martino M., Migliorini F., Paolicchi P., 1997, Icarus, 129, 1

This paper has been typeset from a $\mathrm{T}_{\mathrm{E}} \mathrm{X} / \mathrm{L} \mathrm{T} \mathrm{E} \mathrm{X}$ file prepared by the author. 